\title{
Experimental optimisation of the pitching structural parameters of a fully passive flapping foil turbine ${ }^{\dagger}$
}

\author{
${ }^{\dagger}$ Postprint 2021, Renewable Energy \\ Published version available at https://doi.org/10.1016/j.renene.2021.02.014
}

\author{
Leandro Duarte ${ }^{\mathrm{a}, *}$, Nicolas Dellinger ${ }^{\mathrm{a}}$, Guilhem Dellinger ${ }^{\mathrm{a}, \mathrm{b}}$, Abdellah Ghenaim $^{\mathrm{a}, \mathrm{c}}$, Abdelali Terfous $^{\mathrm{a}, \mathrm{c}}$ \\ ${ }^{a}$ ICube laboratory, department of mechanics, Strasbourg, France \\ ${ }^{b}$ National school for water and environmental engineering, Strasbourg, France \\ ${ }^{c}$ National institute of applied science, Strasbourg, France
}

\begin{abstract}
A reduced scale prototype of a fully passive flapping foil turbine has been designed and tested in a confined channel at a chord Reynolds number of 60000 . Thanks to an original dynamic tuning strategy of the mechanical properties, experiments have been conducted in a wide range of pitching structural parameters for three different pitching axis locations. The best performances have been achieved when the pitching axis is located at one third of the chord length, for which a hydraulic efficiency of $31.9 \%$ has been reached. Relatively good harvesting metrics have also been obtained while moving the pitching axis back to the trailing edge, as long as the pitching stiffness is increased accordingly. The experimental results showed as well that the energy harvested by the pitching motion is negligible compared with the heaving motion. However, a non-zero pitching viscous damping is required in order for the turbine to achieve its best performances.
\end{abstract}

Keywords: fully passive flapping foil; hydrokinetic turbine; micro hydro; fluid-structure interaction; experimental optimisation.

\section{Introduction}

Oscillatory hydrokinetic turbines are innovative devices capable of efficiently harvesting diffuse hydrokinetic energy to locally produce electricity on small-scale. Indeed, microhydro technologies have shown to be a promising solution in the present energy transition scenario, whose primary goal is moving towards a more sustainable development. Besides contributing to the development of the hydropower potential of low current sites (with flow velocities under $1 \mathrm{~ms}^{-1}$ ), such turbines has a limited environmental impact.

The idea of using a two degree of freedom (DOF) oscillating foil as an energy harvesting device has been introduced by McKinney and DeLaurier (1981). They showed that a foil performing a translational motion - heaving - and a rotational motion - pitching - in the cross-section of a flow is capable of harvesting its kinetic energy. Originally, the first concept studied is that of an active flapping foil, in which the two DOF are kinematically constrained in order to enhance energy harvesting. Since then, many numerical and experimental studies have been carried out and successfully proved the feasibility of the concept (Xiao and Zhu, 2014; Young et al., 2014; Wu et al., 2020). Interestingly,

\footnotetext{
* Corresponding author

Email address: leandro.duarte@unistra.fr (Leandro Duarte)
}

Davids (1999) and Kinsey and Dumas (2008) found that the ideal phase shift between heaving and pitching is about $90^{\circ}$. Their numerical studies showed also that the location of pitching axis on the chord line is a key parameter for providing the synchronisation needed for enhancing the turbine performances.

The efficiency of the active flapping foil turbine was experimentally proven by Kinsey et al. (2011) in a two-wing tandem configuration. In their prototype, the pitching axis is located at one third of the chord length from the leading edge. Despite achieving hydraulic efficiencies as high as $40 \%$, more than a quarter of the energy harvested by their prototype was lost in mechanical friction from its complex constraining mechanisms.

An alternative solution introduced by Shimizu et al. (2008) and Zhu et al. (2009) consisted of constraining only the pitching motion and leaving the heaving motion free. The so called semi-passive flapping foil would have the advantage of being less complex from a technological point of view, while proving to be as efficient as the activated devices. However, reports from the first full scale prototype of a semi-passive flapping foil turbine (Stingray, 2002) highlighted prohibitively high maintenance costs related to the pitching activation system.

Finally, the concept of a fully passive flapping foil turbine was introduced by Peng and Zhu (2009). Their nu- 


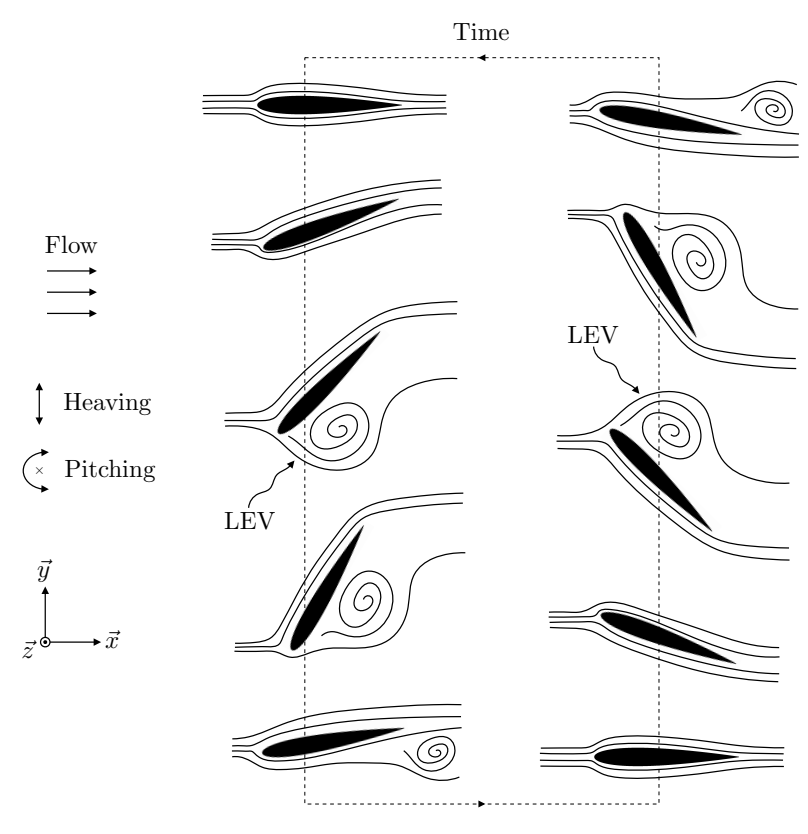

Figure 1: Self-sustained oscillations described by a fully passive flapping foil turbine undergoing deep dynamic stall and shedding a leading edge vortex (LEV).

merical study showed that an elastically mounted foil deprived from any constraining mechanisms could perform self-sustained high amplitude oscillatory motions suitable for energy harvesting. In this configuration, the heaving and pitching motions are completely induced by the fluidstructure interactions. The foil undergoes deep dynamic stall and shed a leading edge vortex (LEV) twice during one period of oscillation. This phenomenon prevents the system from a chaotic behaviour and gives raise to limit cycle oscillations instead. Figure 1 provides a scheme illustrating of the heaving and pitching motions described by a fully passive flapping foil.

The numerical findings of Peng and Zhu (2009) were later refined by Zhu (2012) and Wang et al. (2017). Recently, Duarte et al. (2019) experimentally verified that the dynamic behaviour of the passive flapping foil is highly dependent on the location of the pitching axis and on the pitching stiffness. They provided conditions on those parameters to ensure an appropriate response of the system for energy harvesting purposes.

An extensive numerical optimisation of the structural parameters of a fully passive flapping foil turbine is performed by Veilleux and Dumas (2017). Varying the massspring-damper properties for both DOF of a NACA15 foil with a fixed pitching axis located at one third of the chord length from the leading edge, they found an optimised configuration with a hydraulic efficiency of $33.6 \%$. Those promising numerical results were later verified by Boudreau et al. (2018), who performed the very first experimental study on the subject.

Very recently, Boudreau et al. (2019) conducted a numerical study on a new oscillatory behaviour of a fully passive flapping foil. They found that a high inertia system could operate without undergoing deep dynamic stall and thus without shedding LEV - leading to substantially higher efficiencies. The feasibility of such a heavy flapping foil turbine has yet to be verified experimentally, which is out of scope for the present paper.

In summary, great advances have been achieved both numerically and experimentally on the development of the fully passive flapping foil turbine. Yet relatively little is known about the influence of pitching structural parameters - specially the pitching axis location - on its energy harvesting performances. Indeed, all experiments conducted so far have considered a pitching axis fixed at one third of the chord length, even if it was proven by Peng and Zhu (2009) and Duarte et al. (2019) that a suitable behaviour can be obtained for different configurations.

In such context, this paper presents an experimental study on the influence of pitching structural parameters on the energy harvesting performances of a fully passive flapping foil turbine. Thanks to an original dynamic tuning strategy of the mechanical properties, numerous experiments have been conducted in a wide range of pitching stiffness and pitching viscous damping for three different pitching axis locations.

The paper is structured as follows: Section 2 provides details on the modelling and the harvesting metrics of the turbine, as well as a description of the experimental setup; finally, the results are presented and discussed in Section 3.

\section{Methodology}

\subsection{Turbine modelling and harvesting metrics}

The fully passive flapping foil turbine is modelled by a two DOF damped mass-spring system. The foil interacts with a free stream flow in the $\vec{x}$ direction, performing a heaving motion $y(t)$ in the $\vec{y}$ direction and a pitching motion $\theta(t)$ about the $(P, \vec{z})$ axis, which is parallel to the gravity $\vec{g}$. An upper view kinematic diagram of the model is provided in Figure 2.

The mass, stiffness and viscous damping coefficients related to the heaving motion are referred to as $m_{y}, k_{y}$ and $c_{y}$, respectively; analogously, those related to the pitching motion are referred to as $m_{\theta}, k_{\theta}$ and $c_{\theta}$. The foil has a chord length $c$ and it is elastically mounted at $P$, distant $l_{\theta}$ from the leading edge. The inertial eccentricity $\lambda_{g}$ is the distance from $P$ to the center of gravity of the pitching components $G\left(\lambda_{g}>0\right.$ if $G$ behind $\left.P\right)$. Finally, $I_{\theta}$ is the moment of inertia of the pitching components with respect to $P$. A summary of all physical parameters considered in the model is provided in Table 1.

Limit-cycle oscillations can emerge out of the fluidstructure interactions between the foil and the flow. This particularly interesting behaviour from an energy harvesting perspective is a solution for the equations of motion of the fully passive flapping foil, that can be written: 
Table 1: Physical parameters considered in the modelling of a fully passive flapping foil turbine.

\begin{tabular}{|c|c|c|c|c|}
\hline \multicolumn{4}{|c|}{ Parameter definition } & \multirow{2}{*}{$\begin{array}{l}\text { Non-dimensional form } \\
-\end{array}$} \\
\hline \multirow{3}{*}{$\frac{3}{d}$} & $\rho$ & {$\left[\mathrm{kg} \mathrm{m}^{-3}\right]$} & Fluid density & \\
\hline & $\nu$ & {$\left[\mathrm{m}^{2} \mathrm{~s}^{-1}\right]$} & Fluid kinematic viscosity & - \\
\hline & $U_{\infty}$ & {$\left[\mathrm{m} \mathrm{s}^{-1}\right]$} & Free stream velocity & - \\
\hline \multirow{12}{*}{ 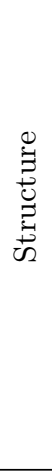 } & $c$ & {$[\mathrm{~m}]$} & Foil chord length & - \\
\hline & $b$ & {$[\mathrm{~m}]$} & Foil span & $b^{*}=b / c$ \\
\hline & $l_{\theta}$ & {$[\mathrm{m}]$} & Pitching axis location & $l_{\theta}^{*}=l_{\theta} / c$ \\
\hline & $\lambda_{g}$ & {$[\mathrm{~m}]$} & Inertial eccentricity & $\lambda_{g}^{*}=\lambda_{g} / c$ \\
\hline & $m_{y}$ & {$[\mathrm{~kg}]$} & Heaving mass & $m_{y}^{*}=m_{y} / \rho b c^{2}$ \\
\hline & $c_{y}$ & {$\left[\mathrm{~N} \mathrm{~s} \mathrm{~m}^{-1}\right]$} & Heaving viscous damping & $c_{y}^{*}=c_{y} / \rho U_{\infty} b c$ \\
\hline & $k_{y}$ & {$\left[\mathrm{~N} \mathrm{~m}^{-1}\right]$} & Heaving stiffness & $k_{y}^{*}=k_{y} / \rho U_{\infty}^{2} b$ \\
\hline & $I_{\theta}$ & {$\left[\mathrm{kg} \mathrm{m}^{2}\right]$} & Moment of inertia & $I_{\theta}^{*}=I_{\theta} / \rho b c^{4}$ \\
\hline & $c_{\theta}$ & {$\left[\mathrm{Nms} \mathrm{rad}^{-1}\right]$} & Pitching viscous damping & $c_{\theta}^{*}=c_{\theta} / \rho U_{\infty} b c^{3}$ \\
\hline & $k_{\theta}$ & {$\left[\mathrm{N} \mathrm{m} \mathrm{rad}^{-1}\right]$} & Pitching stiffness & $k_{\theta}^{*}=k_{\theta} / \rho U_{\infty}^{2} b c^{2}$ \\
\hline & $m_{\theta}$ & {$[\mathrm{kg}]$} & Pitching mass & $m_{\theta}^{*}=m_{\theta} / \rho b c^{2}$ \\
\hline & $\Lambda$ & {$[\mathrm{kg} \mathrm{m}]$} & Static imbalance $\left(\lambda_{g} m_{\theta}\right)$ & $\Lambda^{*}=\Lambda / \rho b c^{3}$ \\
\hline \multirow{6}{*}{ 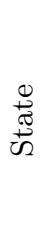 } & $y$ & {$[\mathrm{~m}]$} & Heaving linear position & $y^{*}=y / c$ \\
\hline & $\dot{y}$ & {$\left[\mathrm{~ms}^{-1}\right]$} & Heaving linear velocity & $\dot{y}^{*}=\dot{y} / U_{\infty}$ \\
\hline & $\ddot{y}$ & {$\left[\mathrm{~ms}^{-2}\right]$} & Heaving linear acceleration & $\ddot{y}^{*}=\ddot{y} c / U_{\infty}^{2}$ \\
\hline & $\theta$ & {$[\mathrm{rad}]$} & Pitching angular position & - \\
\hline & $\dot{\theta}$ & {$\left[\operatorname{rad~s}^{-1}\right]$} & Pitching angular velocity & $\dot{\theta}^{*}=\dot{\theta} c / U_{\infty}$ \\
\hline & $\ddot{\theta}$ & {$\left[\mathrm{rad} \mathrm{s}^{-2}\right]$} & Pitching angular acceleration & $\ddot{\theta}^{*}=\ddot{\theta} c^{2} / U_{\infty}^{2}$ \\
\hline
\end{tabular}

$$
\left\{\begin{array}{l}
m_{y} \ddot{y}+c_{y} \dot{y}+k_{y} y+\Lambda\left(\dot{\theta}^{2} \sin \theta-\ddot{\theta} \cos \theta\right)=F_{y} \\
I_{\theta} \ddot{\theta}+c_{\theta} \dot{\theta}+k_{\theta} \theta-\Lambda(\ddot{y} \cos \theta)=M_{\theta}
\end{array}\right.
$$

In equations $1, F_{y}$ stands for the $y$ component of the fluid force over the foil and $M_{\theta}$ for the fluid pitching moment about $(P, \vec{z})$. Besides the implicit coupling provided by the non linearities of the hydrodynamic forces, the static imbalance $\Lambda=m_{\theta} \lambda_{g}$ promotes an explicit non-linear cou-

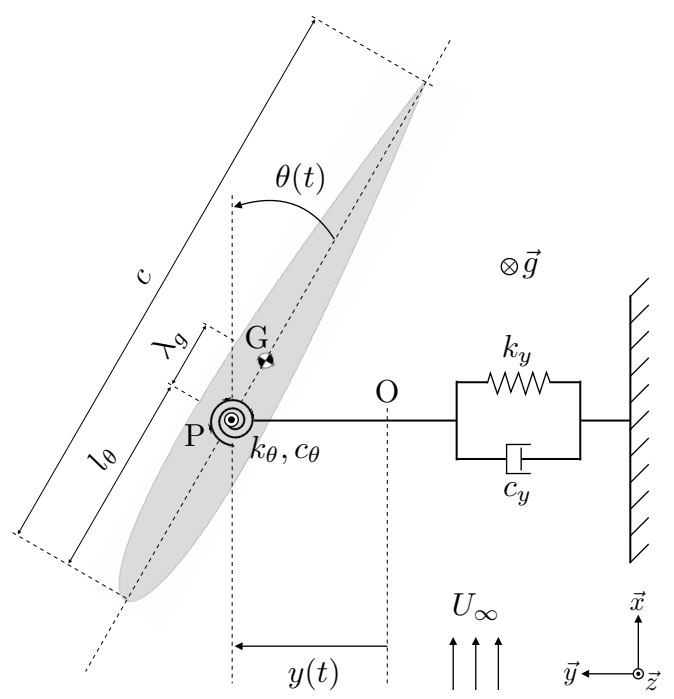

Figure 2: Upper view kinematic diagram of the fully passive flapping foi turbine and its structural parameters. pling between the heaving $y(t)$ and pitching $\theta(t)$ equations.

An energy balance applied to equations 1 shows that only the viscous friction terms produce a non-zero net average power. Indeed, $c_{y}$ and $c_{\theta}$ model the power $P_{e}$ that could be dissipated from the flapping foil in order to produce electricity:

$$
P_{e}(t)=c_{y} \dot{y}^{2}+c_{\theta} \dot{\theta}^{2}
$$

The harvested power $P_{e}$ corresponds to a fraction of the hydraulic power $P_{h}$ available in the cross section of the flow swept by the foil:

$$
P_{h}=\frac{1}{2} \rho U_{\infty}^{3} b d_{y}
$$

with $b$ the foil span and $d_{y}$ the heaving length swept by the foil. Those geometric definitions are illustrated in Figure 3 .

Therefore, the hydraulic efficiency $\eta$ of the turbine is defined by the time average of the ratio between the harvested power $P_{e}$ and the available power $P_{h}$ :

$$
\eta=\frac{1}{\Delta t} \int_{t_{0}}^{t_{0}+\Delta t} \frac{c_{y} \dot{y}^{2}+c_{\theta} \dot{\theta}^{2}}{\frac{1}{2} \rho U_{\infty}^{3} b d_{y}} d t
$$

Alternatively, the harvesting metrics of the fully passive flapping foil turbine can be expressed in terms of the power coefficients $C_{P y}$ and $C_{P \theta}$. They correspond to the nondimensionalised instantaneous power dissipated by the heaving and pitching motions, respectively: 
(a)

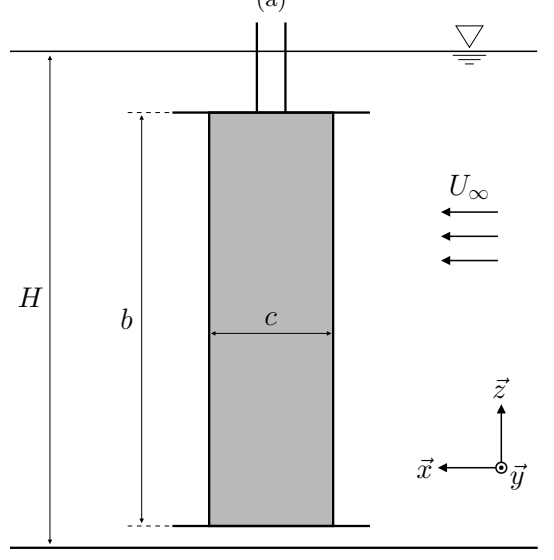

(b)

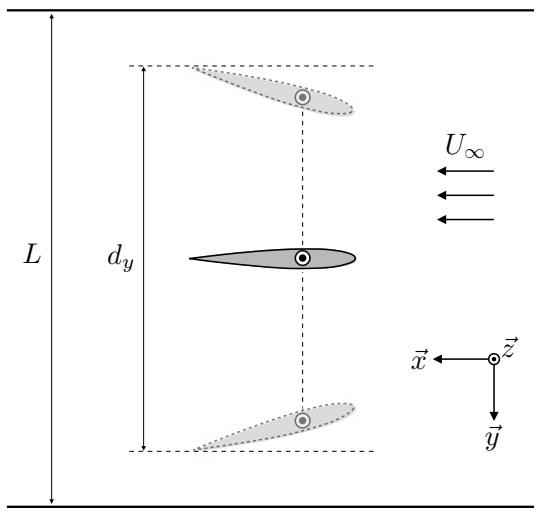

Figure 3: Schematic (a) left side view and (b) top view of a fully passive flapping foil turbine in a confined channel.

$$
\begin{aligned}
& C_{P y}(t)=\frac{c_{y} \dot{y}^{2}}{\frac{1}{2} \rho U_{\infty}^{3} b c} \\
& C_{P \theta}(t)=\frac{c_{\theta} \dot{\theta}^{2}}{\frac{1}{2} \rho U_{\infty}^{3} b c}
\end{aligned}
$$

The total power coefficient $C_{P}$ is obtained by adding equations 5 and 6 , and its time average results in the average power coefficient $\bar{C}_{P}$ :

$$
\begin{gathered}
C_{P}(t)=\frac{c_{y} \dot{y}^{2}+c_{\theta} \dot{\theta}^{2}}{\frac{1}{2} \rho U_{\infty}^{3} b c} \\
\bar{C}_{P}=\frac{1}{\Delta t} \int_{t_{0}}^{t_{0}+\Delta t} C_{P}(t) d t
\end{gathered}
$$

Unlike the hydraulic efficiency, the power coefficients are not scaled with respect to the surface swept by the foil, but rather to its geometric dimensions. As a result, improving the power coefficients of a fully passive flapping foil turbine necessarily implies increasing the harvested power, while the hydraulic efficiency can be improved by reducing the surface swept by the foil.

These coefficients are particularly useful for measuring the contribution of each DOF in the energy harvesting, which is crucial for designing a well suited energy conversion strategy for the electricity production.

\subsection{Experimental setup and study protocol}

A fully passive flapping foil prototype has been designed and tested in a hydraulic channel at the INSA of Strasbourg. A full description of the design process can be found in the PhD thesis of Duarte (2019), Chapter 3. The main components of the experimental setup used in the present work are listed in Figure 4. Pictures of the experimental apparatus are provided in Figure 5.

The turbine prototype consists of a NACA0015 foil of a chord length $c=0.096 \mathrm{~m}$ and a span $b=0.432 \mathrm{~m}$. The foil is tested in a channel wide of $L=0.600 \mathrm{~m}$ and high of $H=0.495 \mathrm{~m}$, leading to a surface blockage ratio given by:

$$
S_{B}=\frac{c b}{L H}=0.14
$$

In order to reproduce the hydraulic conditions of a low current site, the mean free stream velocity in the test section is set to $U_{\infty}=0.625 \mathrm{~m} \mathrm{~s}^{-1}$. Considering the kinematic viscosity of water $\nu=1 \times 10^{-6} \mathrm{~m}^{2} \mathrm{~s}^{-1}$, this leads to a chord Reynolds number defined as:

$$
R e_{c}=\frac{U_{\infty} c}{\nu}=6 \times 10^{4}
$$

The flapping foil prototype is mounted on its shaft through a sliding box allowing for a variable pitching axis location. The pitching shaft casing is mounted on a heaving rail with extension springs. Digital incremental encoders are used to mesure the heaving and pitching motions of the foil, which are linked to electric servomotors through transmission belt systems. The motors are not used to

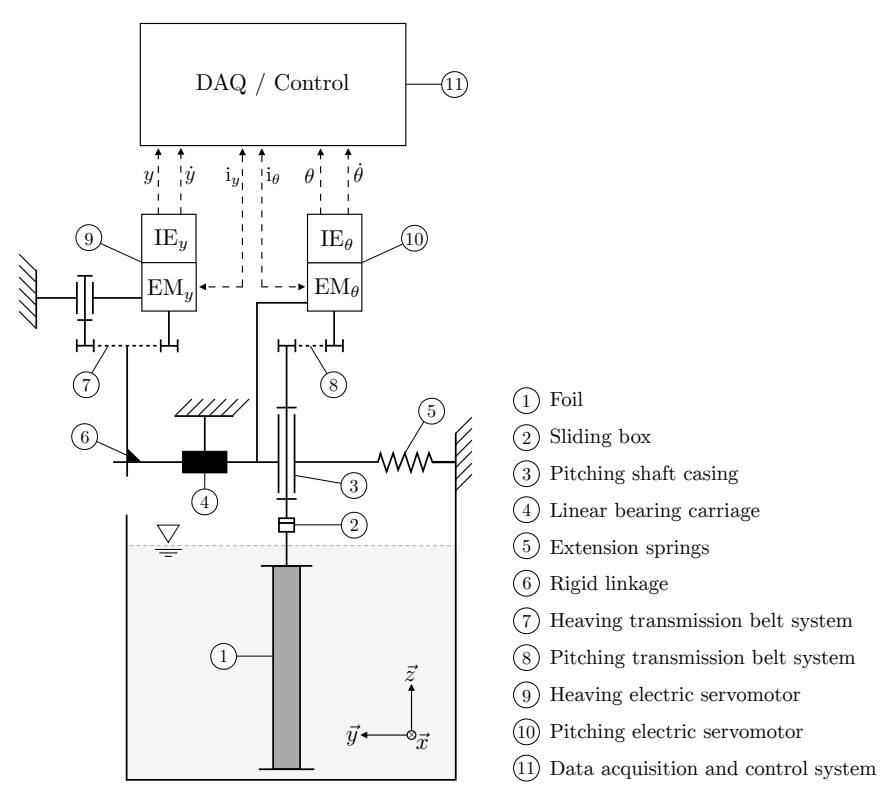

Figure 4: Main components of the experimental setup. 

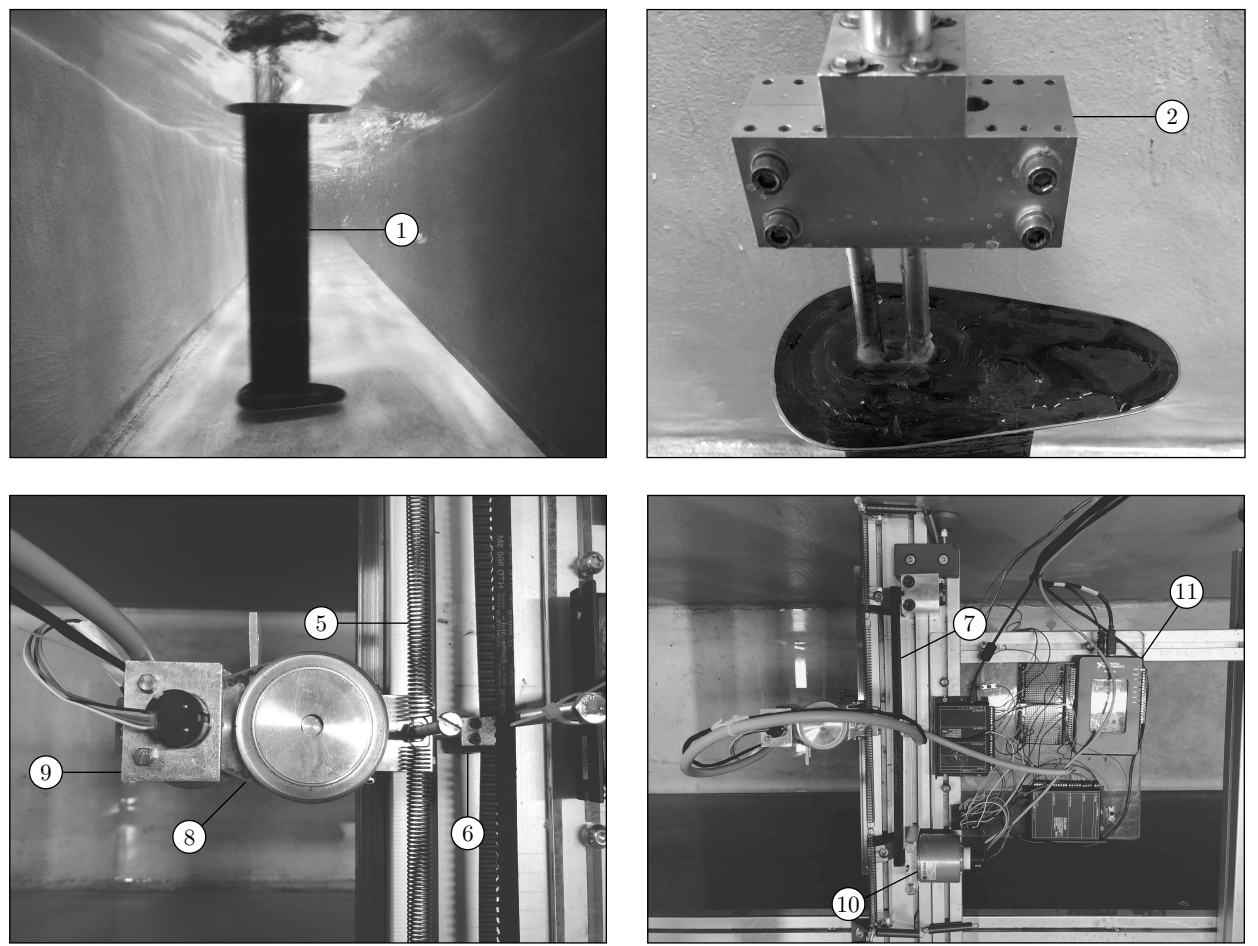

Figure 5: Pictures of the experimental setup of a fully passive flapping foil turbine in a confined channel highlighting its main components (see Figure 4).

constrain the foil motions - indeed, it is a fully passive flapping foil concept. Instead, they are employed in order to artificially modify the mechanical properties of the prototype thanks to an original dynamic tuning strategy (Duarte et al., 2019). This has been particularly useful for introducing the pitching stiffness $k_{\theta}$, getting ideal mechanical linkages by counteracting their inherent friction and setting the viscous friction coefficients $c_{y}$ and $c_{\theta}$ that model the electricity production.

In the present work, the harvesting performances of the turbine will be investigated in the parameter space $k_{\theta}^{*} \times c_{\theta}^{*}$ for three different pitching axis locations $l_{\theta}^{*}$. The structural parameters of the prototype for each configuration is presented in Table 2. A complete description of the methods employed in the characterisation of each mechanical parameter and its corresponding uncertainty is provided by Duarte et al. (2019).

The heaving parameters $\left(m_{y}^{*}, c_{y}^{*}, k_{y}^{*}\right)$ are set according to preliminary results showing that the heaving natural frequency must approach the flapping frequency of the foil (Veilleux and Dumas, 2017; Boudreau et al., 2018; Duarte, 2019). The moment of inertia $I_{\theta}^{*}$ and the static imbalance $\Lambda^{*}$ are slightly different for each configuration since they depend on the pitching axis location. Finally, the ranges of values considered for the pitching viscous damping $c_{\theta}^{*}$ and the pitching stiffness $k_{\theta}^{*}$ were chosen following the response chart provided by Duarte et al. (2019). Indeed, they have stablished necessary conditions on those parameters in order for the foil to describe symmetric high amplitude self-sustained oscillations (which they identified as response type II).

For each experimental test, the kinematics of the foil is recorded during $60 \mathrm{~s}$ and the average harvesting metrics are then computed over 40 oscillations.

\section{Results and discussion}

\subsection{Overall results for the three configurations}

The results in terms of the average power coefficient $\bar{C}_{P}$ and the hydraulic efficiency $\eta$ for the configurations $C_{1}, C_{2}$ and $C_{3}$ are presented in Figure 6. Each test case in the parameter space $k_{\theta}^{*} \times c_{\theta}^{*}$ is identified by a marker. The circle $\circ$ indicates a regular test. The cross $\times$ identifies a test where the heaving amplitude was limited by the available rail length $\left(\left|y^{*}\right|<1.56\right)$. Finally, the square $\square$ is used when a changing in the dynamic behaviour of the foil is observed; in such cases, the foil presented alternative oscillations around two symmetric angles (which has been identified as response type III by Duarte et al. (2019)).

Overall, the best performances of the turbine have been achieved with the pitching axis located at $l_{\theta}^{*}=0.330$ (configuration $C_{1}$ ), supporting the numerical results of Wang et al. (2017). In this first configuration, the energy harvesting is strongly improved by setting a non-zero pitching stiffness $k_{\theta}^{*}$ and viscous damping $c_{\theta}^{*}$. An optimised case is identified at $k_{\theta}^{*}=0.071$ and $c_{\theta}^{*}=0.052$, for which a 
Table 2: Structural parameters of the prototype for the three different configurations considered in the present experimental study.

\begin{tabular}{cccc}
\hline Parameter & $C_{1}$ & $C_{2}$ & $C_{3}$ \\
\hline$l_{\theta}^{*}$ & $0.330 \pm 0.002$ & $0.390 \pm 0.002$ & $0.450 \pm 0.002$ \\
$m_{y}^{*}$ & $0.919 \pm 0.005$ & $0.919 \pm 0.005$ & $0.919 \pm 0.005$ \\
$c_{y}^{*}$ & $0.93 \pm 0.09$ & $0.93 \pm 0.09$ & $0.93 \pm 0.09$ \\
$k_{y}^{*}$ & $0.720 \pm 0.006$ & $0.720 \pm 0.006$ & $0.720 \pm 0.006$ \\
$I_{\theta}^{*}$ & $0.056 \pm 0.002$ & $0.057 \pm 0.002$ & $0.059 \pm 0.002$ \\
$c_{\theta}^{*}$ & {$[0,0.075] \pm 0.002$} & {$[0,0.075] \pm 0.002$} & {$[0,0.075] \pm 0.002$} \\
$k_{\theta}^{*}$ & {$[0,0.090] \pm 0.004$} & {$[0.051,0.206] \pm 0.004$} & {$[0.096,0.174] \pm 0.004$} \\
$\Lambda^{*}$ & $0.0065 \pm 0.0006$ & $-0.0096 \pm 0.0006$ & $-0.0256 \pm 0.0006$ \\
\hline
\end{tabular}

power coefficient of $\bar{C}_{P}=1.10$ and a hydraulic efficiency of $\eta=31.9 \%$ could be measured. A video of the prototype operating in the optimised configuration is provided with the online version of this paper.

When the stiffness $k_{\theta}^{*}$ approaches the maximum values, there is a steep drop in the harvesting performances because of the change in the dynamic behaviour of the foil. Indeed, the kinematic response observed for $k_{\theta}^{*}>0.09$ in configuration $C_{1}$ can be identified as a response III according to Duarte et al. (2019): the foil motion is irregular and switches between two oscillating states, which is not suitable for energy harvesting purposes.

Moving the pitching axis back to $l_{\theta}^{*}=0.390$ (configuration $C_{2}$ ), high amplitude self sustained oscillations could be observed as long as the pitching stiffness was adjusted accordingly $\left(0.06<k_{\theta}^{*}<0.20\right)$. Just as in configuration $C_{1}$, the best performances achieved in configuration $C_{2}$ require relatively high values of pitching stiffness $k_{\theta}^{*}$ and viscous damping $c_{\theta}^{*}$. As will be discussed in Section 3.2, those parameters help slowing down the pitching motion of the foil and enhance the heaving amplitudes.

An optimised case could be identified in configuration $C_{2}$ at $k_{\theta}^{*}=0.167$ and $c_{\theta}^{*}=0.061$, with an average power coefficient of $\bar{C}_{P}=1.08$ and a hydraulic efficiency of $\eta=$ $31.1 \%$. However, the experimental results suggest that even better performances could be achieved for $k_{\theta}^{*}>0.18$ if the heaving amplitude was not limited by the available rail length.

Finally, moving the pitching axis further back to $l_{\theta}^{*}=$ 0.450 (configuration $C_{3}$ ), the energy harvesting metrics of the prototype drop considerably. Unlike for configurations $C_{1}$ and $C_{2}$, high values of pitching viscous damping $c_{\theta}^{*}$ in configuration $C_{3}$ triggers a change in the dynamic behaviour of the foil. In fact, an unsuitable kinematics (response III) has been observed for roughly half the experimental tests in this configuration. Relatively good performances could still be identified at $k_{\theta}^{*}=0.174$ and $c_{\theta}^{*}=0.056$, with a power coefficient of $\bar{C}_{P}=0.67$ and a hydraulic efficiency of $\eta=20.2 \%$. It can be assumed that better performances would have been achieved in configuration $C_{3}$ for higher values of pitching stiffness $k_{\theta}^{*}$. This assumption could not be experimentally verified because of the limited power of the pitching electric servomotor.

A more detailed analysis of the experimental results for the configuration $C_{1}$ - for which the best performances have been achieved - is provided in what follows. A complet analysis for all configurations can be found in the $\mathrm{PhD}$ thesis of Duarte (2019), Chapter 5.

\subsection{Detailed analysis of the configuration $C_{1}$}

It is with the pitching axis located at $l_{\theta}^{*}=0.330$ that the fully passive flapping foil prototype has shown its best energy harvesting performances in the present study. The optimised case identified for the configuration $C_{1}$ lies in a fairly wide area of the parameter space $k_{\theta}^{*} \times c_{\theta}^{*}$ where the turbine is very efficient. Moreover, it can be noted from Figure 6 that the hydraulic efficiency $\eta$ is much less sensitive to the pitching stiffness $k_{\theta}^{*}$ than the average power coefficient $\bar{C}_{P}$. In fact, the turbine is equally efficient in the whole range of $k_{\theta}^{*}$ as long as the viscous damping $c_{\theta}^{*}$ is sufficiently high. This suggests that, along with the decrease observed in the harvested power at a low pitching stiffness, the surface swept by the foil decreases as well, so that the hydraulic efficiency remains constant.

Additional information on the evolution of the harvesting metrics for configuration $C_{1}$ around the optimised case is provided in Figure 7 . For both the average power coefficient $\bar{C}_{P}$ and the hydraulic efficiency $\eta$, the error bars represent the measurement uncertainty inherited from the characterisation procedure of the mechanical friction between the linear bearing carriage and the heaving rail (Duarte et al., 2019). In spite of that, the optimised case at $k_{\theta}^{*}=0.071$ and $c_{\theta}^{*}=0.052$ can be easily identified from the average power coefficient plots in Figure 7.

The kinematics and harvesting metrics of the optimised case are compared to those of an initial case without any pitching stiffness or viscous damping $\left(k_{\theta}^{*}=0, c_{\theta}^{*}=0\right)$. The comparison results are summarised in Table 3 . The nondimensionalised heaving amplitude $A_{y}^{*}$ - defined by the heaving amplitude $A_{y}$ normalised by the chord length $c$ - considerably increases in the optimised case, while the pitching amplitude $A_{\theta}$ is slightly lower. It can be inferred that a more stiff pitching system enhances the energy harvesting by reducing the pitching angles of the foil and thus delaying the dynamic stall. 

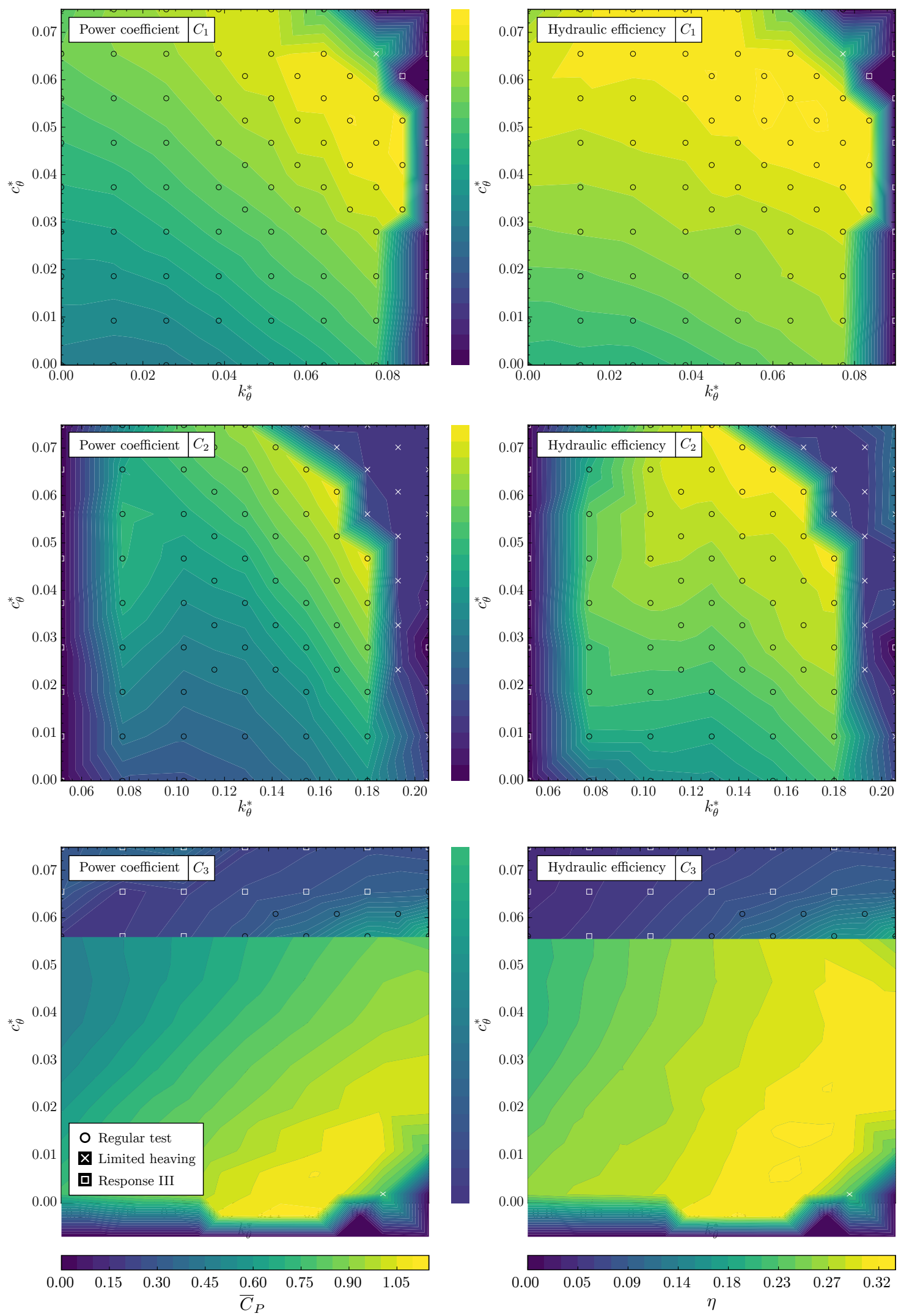

Figure 6: Energy harvesting performances of the prototype in the parameter space $k_{\theta}^{*} \times c_{\theta}^{*}$ for the configurations $C_{1}, C_{2}$ and $C_{3}$ in terms of (left) the average power coefficient $\bar{C}_{P}$ and (right) the hydraulic efficiency $\eta$. 
(a)

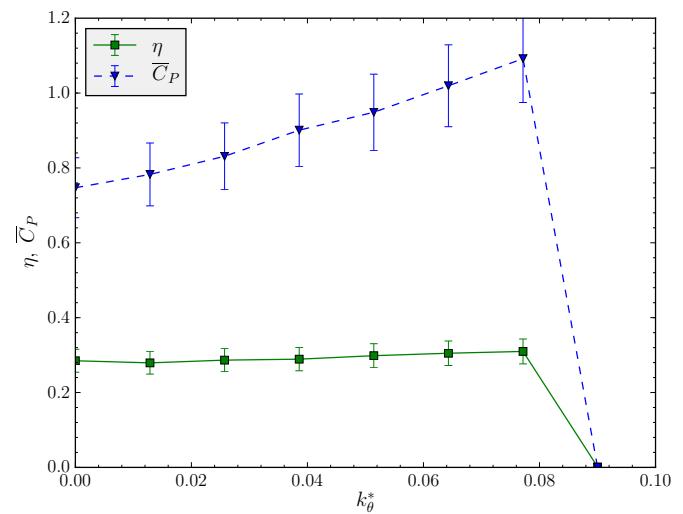

(b)

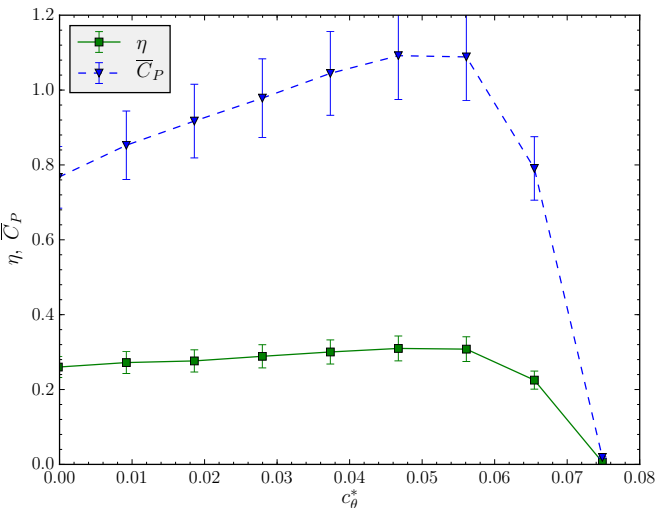

Figure 7: Plots of the averaged power coefficient $\bar{C}_{P}$ and hydraulic efficiency $\eta$ with error bars for (a) a constant pitching viscous damping $c_{\theta}^{*}=0.052$ and (b) a constant pitching stiffness $k_{\theta}^{*}=0.071$, with the pitching axis located at $l_{\theta}^{*}=0.330\left(\right.$ configuration $\left.C_{1}\right)$.

The flapping frequency of the turbine $f$ - expressed in terms of the reduced frequency $f^{*}$ as defined in equation 11 - marginally decreases during the optimisation process. The reduced frequency for the optimised case is very close to that of the fully passive flapping foil prototype studied by Boudreau et al. (2018). In addition, it fits the prescribed range of values (from 0.1 to 0.2 ) for achieving the best performances with a fully constrained flapping foil turbine (Kinsey and Dumas, 2014; Xiao and Zhu, 2014; Young et al., 2014), as expected.

$$
f^{*}=\frac{f c}{U_{\infty}}
$$

The improvement of the harvesting performances of the turbine while optimising its pitching structural parameters are summarised as well in Table 3. The average power coefficient $\bar{C}_{P}$ more than doubled and a significant increase is also observed for the hydraulic efficiency $\eta$. To provide a better understanding of how such significant enhancement is achieved, the kinematics and instantaneous power coefficients of the fully passive flapping foil turbine are presented in Figure 8 for both initial and optimised cases.

It can be verified from the temporal evolution of the foil kinematics over $60 \mathrm{~s}$ of recorded data that the heaving amplitude greatly increases in the optimised case. A sample

Table 3: Kinematic and harvesting metrics of the fully passive flapping foil prototype for the initial case $\left(k_{\theta}^{*}=0, c_{\theta}^{*}=0\right)$ and the optimised case $\left(k_{\theta}^{*}=0.071, c_{\theta}^{*}=0.052\right)$ with the pitching axis located at $l_{\theta}^{*}=0.330$ (configuration $C_{1}$ ).

\begin{tabular}{cccc}
\hline Metric & Initial case & Optimised case & Variation \\
\hline$A_{y}^{*}$ & 0.83 & 1.36 & $63.9 \%$ \\
$A_{\theta}$ & $96^{\circ}$ & $76^{\circ}$ & $-20.8 \%$ \\
$f^{*}$ & 0.148 & 0.131 & $-11.5 \%$ \\
$\bar{C}_{P}$ & 0.47 & 1.10 & $134.0 \%$ \\
$\eta$ & $20.4 \%$ & $31.9 \%$ & $56.4 \%$ \\
\hline
\end{tabular}

of one oscillation period of the foil is also provided in Figure 8 , where $t^{*}=t / T$ is the normalised time with respect to the turbine period of oscillation $T$. Those plots allows for measuring the phase shift $\phi$ between heaving and pitching motions, which grows from $60^{\circ}$ to $76^{\circ}$ in the optimised case and thus approaches the ideal value of $90^{\circ}$ (Davids, 1999; Kinsey and Dumas, 2008).

Moreover, it can be noted that the pitching velocity considerably decreases around $t^{*}=0.22$ and $t^{*}=0.72$ in the optimised case, keeping the foil at lower pitching angles. Thanks to that, the foil reaches higher heaving velocities and therefore the turbine shows better harvesting performances, as it can be seen from the evolution of the instantaneous power coefficients during one period of oscillation. Indeed, a $C_{P}$ as high as 2.5 can be observed in the optimised case, while it remains below 1.5 in the initial case.

Finally, the instantaneous power coefficients plotted in Figure 8 show as well the contribution of each DOF in the energy harvesting. In the initial case, the total power is provided only by the heaving motion since no energy is dissipated in the pitching motion $\left(c_{\theta}^{*}=0 \Rightarrow C_{P \theta}=0\right)$. By introducing a $c_{\theta}^{*}>0$ in the optimised case, a small contribution from the pitching motion of about $8 \%$ of the total energy harvested can be measured. As a result, it can be stated that a non-zero pitching viscous damping in the optimised case indirectly enhances the energy harvesting in heaving. In practice, the amount of $c_{\theta}^{*}$ needed for achieving the higher performances of the turbine could be introduced only by mechanical friction, given that the contribution of $C_{P \theta}$ to the energy harvesting is irrelevant.

\section{Conclusion}

An experimental optimisation of the pitching structural parameters of a fully passive flapping foil turbine prototype has been conducted in a confined channel at a chord Reynolds number of $6 \times 10^{4}$. The turbine prototype consists essentially of a NACA0015 foil elastically mounted on 

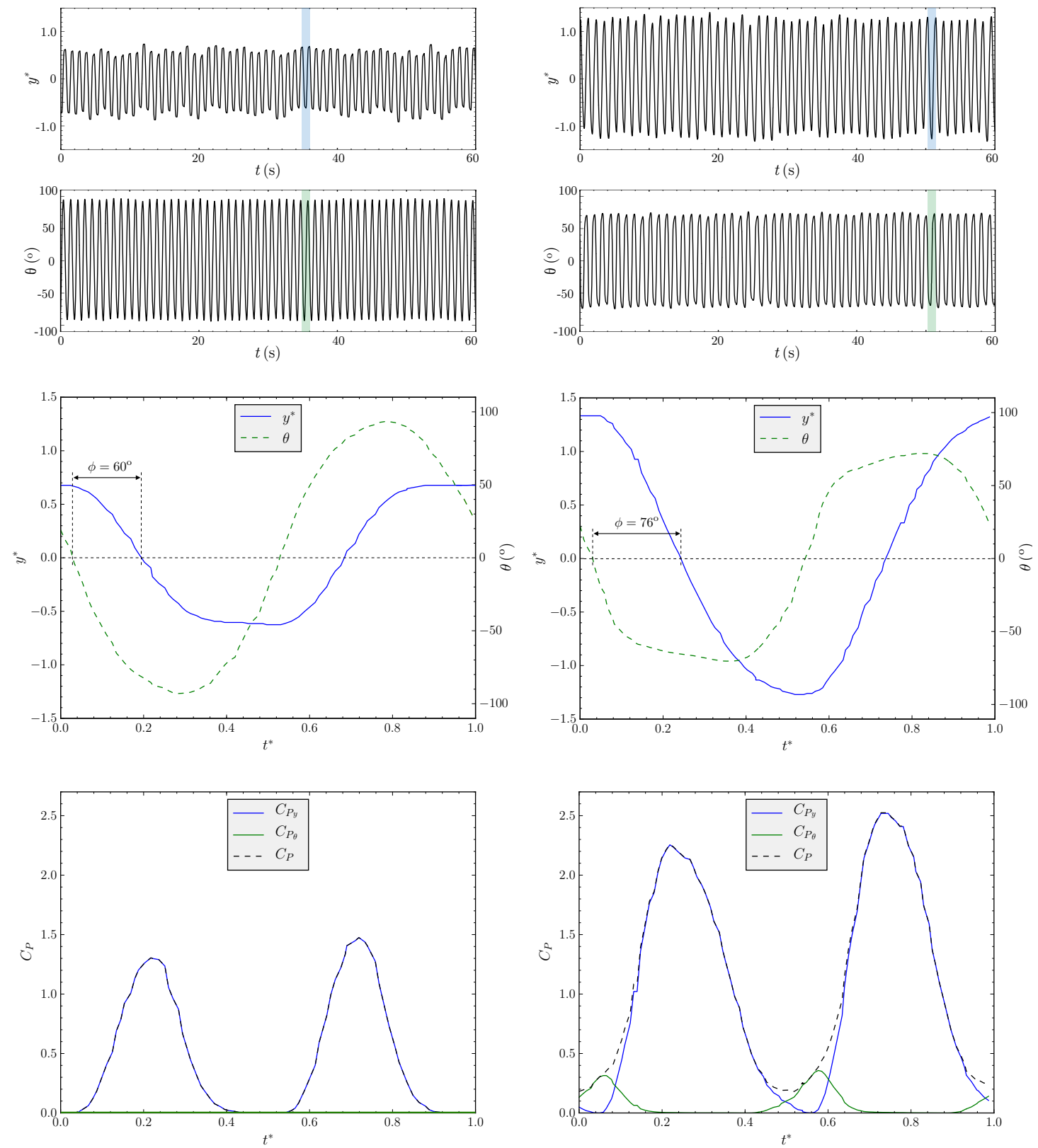

Figure 8: Kinematics and instantaneous power coefficients of the fully passive flapping foil prototype with the pitching axis located at $l_{\theta}^{*}=0.330$ (configuration $\left.C_{1}\right)$ for $(\mathrm{a})$ the initial case $\left(k_{\theta}^{*}=0, c_{\theta}^{*}=0\right)$ and $(\mathrm{b})$ the optimised case $\left(k_{\theta}^{*}=0.071, c_{\theta}^{*}=0.052\right)$.

a pitching shaft and a heaving rail. Electric servomotors are employed in an original dynamic tuning strategy allowing for artificially changing the mechanical properties of the turbine. Thanks to that, the energy harvesting performances of the prototype could be investigated in a wide range of pitching stiffness $k_{\theta}^{*}$ and pitching viscous damping $c_{\theta}^{*}$ for three different pitching axis locations $l_{\theta}^{*}$.

Overall, the best performances of the turbine have been achieved with the pitching axis located at one third of the chord length $\left(l_{\theta}^{*}=0.330\right)$. In this first configuration, an optimised case have been identified at $k_{\theta}^{*}=0.071$ and $c_{\theta}^{*}=$ 0.052 , for which an average power coefficient of $\bar{C}_{P}=1.10$ and a hydraulic efficiency of $\eta=31.9 \%$ could be measured. Moving the pitching axis back to the trailing edge required some adjustments in the pitching stiffness in order for the self-sustained high amplitude oscillations of the foil to be preserved. Comparable performances have been reached with a pitching axis located at $l_{\theta}^{*}=0.390$, but the energy harvesting considerably drops when the pitching axis is moved further back to the trailing edge $\left(l_{\theta}^{*}=0.450\right)$.

The improvements in the energy harvesting of the prototype while optimising its pitching structural parameters are achieved through significant changes in the foil kinematics. In the optimised case, the pitching amplitude decreases, 
delaying the dynamic stall and allowing for higher heaving amplitudes. Besides, the phase shift between heaving and pitching motions is increased, approaching the ideal value of $90^{\circ}$. It has been noted as well that the oscillation frequency of the prototype is close to the values prescribed in the literature for achieving the best performances with an active flapping foil turbine.

Moreover, it has been found that the share of the pitching motion in the energy harvesting is relatively small when compared to the heaving motion. However, a non-zero pitching viscous damping is still required for the turbine to achieve its best performances. Those results support the design of a fully passive flapping foil turbine where only the heaving motion is engaged in the electricity production.

The influence of other structural parameters - such as the static imbalance or the moment of inertia - on the harvesting performances of the turbine has yet to be studied. It could be considered as well adapting the prototype in order to reduce the blockage ratio and introduce higher values of pitching stiffness. This would allow for a more refined investigation of the configurations with the pitching axis located downstream from one third of the chord length. Future work should also address the sensitivity of the harvesting performances to fluctuations in hydraulic conditions and the strategies to convert the mechanical power of the flapping foil into electricity.

\section{Acknowledgements}

This research project is supported by University of Strasbourg, ICube Laboratory and INSA Strasbourg. The authors would like to show their gratitude to the colleagues from the lab who provided valuable insight and expertise that greatly assisted the research.

\section{References}

Boudreau, M., Dumas, G., Rahimpour, M., and Oshkai, P. (2018). Experimental investigation of the energy extraction by a fullypassive flapping-foil hydrokinetic turbine prototype. Journal of Fluids and Structures, 82:446 - 472 .

Boudreau, M., Picard-Deland, M., and Dumas, G. (2019). A parametric study and optimization of the fully-passive flapping-foil turbine at high reynolds number. Renewable Energy, 146.

Davids, S. T. (1999). A computational and experimental investigation of a flutter generator. Master's thesis, Naval postgraduate school.

Duarte, L. (2019). Conception et optimisation d'un système hydrolien à aile oscillante passive. $\mathrm{PhD}$ thesis, ICube Laboratory, University of Strasbourg. Under the supervision of A. Terfous, N. Dellinger and G. Dellinger.

Duarte, L., Dellinger, N., Dellinger, G., Ghenaim, A., and Terfous, A. (2019). Experimental investigation of the dynamic behaviour of a fully passive flapping foil hydrokinetic turbine. Journal of Fluids and Structures, $88: 1-12$.

Kinsey, T. and Dumas, G. (2008). Parametric study of an oscillating airfoil in power extraction regime. Aiaa Journal - AIAA J, 46:13181330 .

Kinsey, T. and Dumas, G. (2014). Optimal operating parameters for an oscillating foil turbine at reynolds number 500,000. AIAA Journal, 52:1885-1895.
Kinsey, T., Dumas, G., Lalande, G., Ruel, J., Méhut, A., Viarouge, P., Lemay, J., and Jean, Y. (2011). Prototype testing of a hydrokinetic turbine based on oscillating hydrofoils. Renewable Energy, 36(6):1710 - 1718 .

McKinney, W. and DeLaurier, J. (1981). Wingmill: An oscillatingwing windmill. Journal of Energy, 5(2):109-115.

Peng, Z. and Zhu, Q. (2009). Energy harvesting through flow-induced oscillations of a foil. Physics of Fluids, 21(12):123602.

Shimizu, E., Isogai, K., and Obayashi, S. (2008). Multiobjective design study of a flapping wing power generator. Journal of Fluids Engineering-transactions of The Asme - J FLUID ENG, 130.

Stingray (2002). Research and development of a $150 \mathrm{kw}$ tidal stream generator. Technical report, Engineering Business Ltd.

Veilleux, J.-C. and Dumas, G. (2017). Numerical optimization of a fully-passive flapping-airfoil turbine. Journal of Fluids and Structures, 70:102-130.

Wang, Z., Du, L., Zhao, J., and Sun, X. (2017). Structural response and energy extraction of a fully passive flapping foil. Journal of Fluids and Structures, 72:96 - 113.

Wu, X., Zhang, X., Tian, X., Li, X., and Lu, W. (2020). A review on fluid dynamics of flapping foils. Ocean Engineering, 195:106712.

Xiao, Q. and Zhu, Q. (2014). A review on flow energy harvesters based on flapping foils. Journal of Fluids and Structures, 46:174-191.

Young, J., Lai, J. C., and Platzer, M. F. (2014). A review of progress and challenges in flapping foil power generation. Progress in Aerospace Sciences, 67:2 - 28.

Zhu, Q. (2012). Energy harvesting by a purely passive flapping foil from shear flows. Journal of Fluids and Structures, 34:157-169.

Zhu, Q., Haase, M., and Wu, C. (2009). Modeling the capacity of a novel flow-energy harvester. Applied Mathematical Modelling APPL MATH MODEL, 33:2207-2217. 